\title{
Phosphoproteomics identifies potential downstream targets of the integrin $\alpha 2 \beta 1$ inhibitor BTT-3033 in prostate stromal cells
}

\author{
Bingsheng Li ${ }^{1,2 \#}$, Pan $\mathrm{Li}^{3 \#}$, Weiping Xia ${ }^{1}$, Baiyang You ${ }^{4,5}$, Qingfeng Yu ${ }^{6}$, Bo Zhang ${ }^{1}$, Ru Huang ${ }^{2}$, \\ Ruixiao Wang ${ }^{2}$, Yuhan $\mathrm{Liu}^{2}$, Zhi Chen ${ }^{1}$, Yu Gan ${ }^{1}$, Yao He ${ }^{1}$, Martin Hennenberg ${ }^{2}$, Christian G. Stief ${ }^{2}$, \\ Xiang Chen ${ }^{1}$
}

${ }^{1}$ Department of Urology, Xiangya Hospital, Central South University, Changsha, China; ${ }^{2}$ Department of Urology, University Hospital Munich, LMU Munich, Munich, Germany; ${ }^{3}$ Department of Pathology, LMU Munich, Munich, Germany; ${ }^{4}$ National Clinical Research Center for Geriatric Disorders, Xiangya Hospital, Central South University, Changsha, China; ${ }^{5}$ Division of Cardiac Rehabilitation, Department of Physical Medicine and Rehabilitation, Xiangya Hospital, Central South University, Changsha, China; ${ }^{6}$ Department of Urology and Guangdong Key Laboratory of Urology, The First Affiliated Hospital of Guangzhou Medical University, Guangzhou, China

Contributions: (I) Conception and design: Y He, B Li, P Li, M Hennenberg; (II) Administrative support: X Chen, CG Stief; (III) Provision of study materials or patients: X Chen; (IV) Collection and assembly of data: B Li, P Li, W Xia, B You; (V) Data analysis and interpretation: P Li, B Li; (VI) Manuscript writing: All authors; (VII) Final approval of manuscript: All authors.

\#These authors contributed equally to this work.

Correspondence to: Dr. Yao He. Department of Urology, Xiangya Hospital, Central South University, No. 87 Xiangya Road, Changsha 410008, China. Email: heyao1984@163.com.

Background: Integrin $\alpha 2 \beta 1$ inhibitor BTT-3033 (1-(4-fluorophenyl)-N-methyl-N-[4[[(phenylamino) carbonyl]amino]phenyl]-1H-pyrazole-4-sulfonamide) was recently reported to inhibit neurogenic and thromboxane A2-induced human prostate smooth muscle contraction, and thus represents a target with a different inhibition spectrum than that of $\alpha 1$-blockers in benign prostate hyperplasia (BPH) treatments. Clarifying the underlying mechanisms of the inhibition effects will provide insights into the role of integrin $\alpha 2 \beta 1$ in prostate contraction and enable new intracellular targets for smooth muscle contraction to be explored.

Methods: ProteomeHD was used to predict and enrich the top co-regulated proteins of integrin $\alpha 2$ (ITGA2). A phosphoproteomic analysis was conducted on human prostate stromal cells (WPMY-1) treated with 1 or $10 \mu \mathrm{M}$ of BTT-3033 or solvent for controls. A clustering analysis was conducted to identify the intracellular targets that were inhibited in a dose-dependent manner. Gene ontology (GO) and annotation enrichments were conducted to examine any functional alterations and identify possible downstream targets. A Kinase-substrate enrichment analysis (KSEA) was conducted to identify kinases-substrate relationships.

Results: Enrichments of the actin cytoskeleton and guanosine triphosphatases (GTPases) signaling were predicted from the co-regulated proteins with ITGA2. LIM domain kinases, including LIM domain and actin-binding 1 (LIMA1), zyxin (ZYX), and thyroid receptor-interacting protein 6 (TRIP6), which are functionally associated with focal adhesions and the cytoskeleton, were present in the clusters with dosedependent phosphorylation inhibition pattern. 15 substrates were dose-dependently inhibited according to the KSEA, including polo-like kinase 1 (PLK1), and GTPases signaling proteins, such as disheveled segment polarity protein 2 (DVL2).

Conclusions: In this study, we proposed that the mechanisms underlying the contractile and proliferative effects of integrin $\alpha 2 \beta 1$ are the LIM domain kinases, including the ZYX family, and substrates, including PLK1 and DVL2.

Keywords: Integrin $\alpha 2 \beta 1$; BTT-3033; benign prostatic hyperplasia (BPH); lower urinary tract symptoms (LUTS); phosphoproteomics

\footnotetext{
^ ORCID: 0000-0003-0045-3192.
} 
Submitted Jun 09, 2021. Accepted for publication Aug 30, 2021.

doi: 10.21037/atm-21-3194

View this article at: https://dx.doi.org/10.21037/atm-21-3194

\section{Introduction}

Male lower urinary tract symptoms (LUTS) that are suggestive of benign prostate hyperplasia (BPH) are the result of a mechanical obstruction arising from an enlarged prostate and dynamic factors, such as prostate smooth muscle tone (1). The proliferation of prostate stromal cells has been reported to contribute significantly to $\mathrm{BPH}$, especially symptomatic BPH (2). The tone of the prostate smooth muscle located in the prostatic stromal is also increased in BPH (3). BTT-3033 (1-(4-fluorophenyl)$\mathrm{N}$-methyl-N-[4[[(phenylamino)carbonyl]amino]phenyl]$1 \mathrm{H}$-pyrazole-4-sulfonamide) is an integrin $\alpha 2 \beta 1$ inhibitor that has recently been shown to inhibit neurogenic and thromboxane A2-induced prostate smooth muscle contraction in the human prostate at a concentration of $1 \mu \mathrm{M}$, and thus represents a candidate target for $\mathrm{BPH}$ treatments with a different inhibition spectrum than those of $\alpha 1$-blockers (4). It has been suggested that thromboxane A2 may induce smooth muscle contraction by mechanisms other than endothelin-1 or adrenergic agonists; however, the mechanisms by which this occurs are still far from being understood. Thus, we sought to investigate the mechanisms underlying the contraction inhibition caused by BTT-3033, and determine whether BTT-3033 affects the proliferation of prostate stromal cells.

In mammals, a total of 24 different integrin heterodimers are formed by non-covalent bonds between $18 \alpha$-subunits and $8 \beta$-subunits (5). Integrins mediate adhesion between cells and the extracellular matrix (ECM) (5). Distribution of the integrin subtype and corresponding functions is diverse in different organs due to various cell types and the distinct ECM components. This relatively specific feature may be able to provide high selectivity and therapeutic approaches with limited side effects, and thus has promising practical applications. Integrin $\alpha 2 \beta 1$, a collagen-I-specific receptor that attaches stromal cells to the ECM, mediates multiple cell functions (6-8). The cytoplasmic part of integrin $\alpha 2 \beta 1$ is the center for focal adhesion (FA) (9) assembly, with the assembling partners including the Src family kinase (SFK), focal adhesion kinase (FAK) and integrin-linked kinase (ILK) of which the modifications may affect biological processes $(4,10,11)$. We conducted a preliminary test (data not shown) that suggested that SFK, FAK, and ILK may not be the downstream targets of BTT-3033 at $1 \mu \mathrm{M}$ in the context of contraction inhibition. These preliminary results prompted the consecutive investigations reported in this article. In this study, we investigated the effects of integrin inhibitor BTT-3033 on the proliferation of the prostate stromal cell line WPMY-1, predicted the top co-regulated proteins of integrin $\alpha 2 \beta 1$, and conducted a phosphoproteomics analysis to identify the potential mechanisms of effects of BTT-3033 on prostate stromal cells. We present the following article in accordance with the MDAR reporting checklist (available at https://dx.doi.org/10.21037/atm-21-3194).

\section{Methods}

\section{Cell culture}

We purchased WPMY-1 (catalog no. CRL-2854), an immortalized cell line from human prostate stroma, without any evidence of malignancy, from American Type Culture Collection (Manassas, VA, USA). WPMY-1 cells show characteristics of myofibroblasts and prostate smooth muscle cells, including the expression of vimentin, $\alpha$-smooth muscle actin, calponin and $\alpha_{1 \mathrm{~A}}$-adrenoceptors, but lack the expression of cytokeratin and tyrosine hydroxylase (12). Consequently, WPMY-1 is commonly used to investigate mechanisms of smooth muscle contraction and cell proliferation (12-14). The WPMY-1 cells were grown in Dulbecco's Modified Eagle Medium (Biological Industries, USA) supplemented with $10 \%$ fetal bovine serum and $1 \%$ penicillin/streptomycin at $37{ }^{\circ} \mathrm{C}$ with $5 \%$ carbon dioxide The WPMY-1 cells were randomly grouped and treated with solvent, and BTT-3033 at different concentrations, including $1 \mu \mathrm{M}$ in the context of smooth muscle contraction inhibition, and $10 \mu M$ in the context of cell cycle arrest.

\section{Proteins co-regulation prediction}

ProteomeHD (15), an online proteome-wide co-regulation analysis tool, was used to identify the proteins that were co-regulated with the $\alpha 2$ integrin subunit (ITGA2) of the proteasome. It should be noted that unlike the $\beta 1$ integrin subunit (ITGB1), ITGA2 only assembles one kind 
of integrin heterodimer, i.e. $\alpha 2 \beta 1$. Enrichment analysis of KEGG pathways and gene ontology (GO) biological processes was undertaken via proteomeHD using the selected proteins.

\section{alamarBlue assay}

Cell viability was assessed using an alamarBlue assay. The WPMY-1 cells were seeded into 96-well plates $(1,000$ cells $/ 100 \mu \mathrm{L}$ ), and treated with $0.3,1,3$, or $10 \mu \mathrm{M}$ BTT3033 or dimethyl sulfoxide (DMSO) overnight. An alamarBlue (Bio-Rad, UK) solution was then added to each well, and the cells were incubated for 3-4 hours at $37{ }^{\circ} \mathrm{C}$. Subsequently, absorbance as an indicator of cell viability was measured with an excitation at $570 \mathrm{~nm}$ and an emission at $590 \mathrm{~nm}$ using Varioskan (Thermo) luminometer at 0 , 24, 48, and 96 h. Readouts were saved as Excel files and transferred into Prism Graphpad (8.4.3) for analysis.

\section{Protein sample preparation}

Samples of WMPY-1 from the BTT-3033 low $(1 \mu \mathrm{M})$ and high $(10 \mu \mathrm{M})$ groups and the control group were lysed in lysis buffer $\left(100 \mathrm{mM} \mathrm{NH}_{4} \mathrm{HCO}_{3}, 6 \mathrm{M}\right.$ urea, and $0.2 \% \mathrm{SDS}$, $\mathrm{pH}=8$ ) with a phosphatase inhibitor (phosSTOP, Roche, 1 tablet per $10 \mathrm{~mL}$ ). The enrichment of phosphopeptide was carried out using PHOS-Select ${ }^{\mathrm{TM}}$ Iron Affinity Gel (Sigma, P9740). Lyophilized powder was dissolved and centrifuged after which the supernatant was loaded to the IMAC-Fe column, and the collection was centrifuged and eluted twice before being lyophilized.

\section{Liquid chromatography with tandem mass spectrometry}

The lyophilized powder was dissolved and centrifuged, after which $1 \mu \mathrm{g}$ supernatant was used for detection. Bottom-up proteomics analyses were performed in an EASY-nLC ${ }^{\mathrm{TM}}$ 1200 UHPLC system (Thermo Fisher) coupled with an Orbitrap Q Exactive HF-X mass spectrometer (Thermo Fisher) in the data-dependent acquisition mode. The separated peptides were analyzed by Q Exactive HF-X with an ion source of Nanospray Flex ${ }^{\mathrm{TM}}$ (ESI).

\section{The identification and quantitation of protein}

The resulting spectra from each sample were searched separately against the homo_sapiens_uniprot_2020.07.02. fasta (192283 sequences) database by Proteome Discoverer
2.4 (PD 2.4, thermo) with false discovery rate (FDR) $<1 \%$ at the level of proteins and peptides.

\section{Analyses of motif and kinase-substrate relationships}

The motif-x algorithm was used to identify the motifs enriched within a set of phosphate sites. All enrichments were carried out for 7 residues surrounding the central residue with occurrences $>20$ and $\mathrm{P}<10^{-6}$. All identified serine, threonine, and tyrosine phosphorylation sites were scored and calculated using a Kinase-substrate enrichment analysis (KSEA) (16).

\section{Bioinformatic analysis}

All the identified proteins were assigned their gene symbol via UniProtKB (http://www.uniprot.org/). A heatmap and clustering analysis based on the expression/modification of proteins or phosphorylation sites was conducted and visualized using $\mathrm{R}$ (the "pheatmap" package) (17) according to the (phospho-) proteomic assay. A protein-protein interaction (PPI) and enrichments of GO and annotation analyses were performed using STRING (https://string-db. org/) (18), and visualized using Cytoscape (Version 3.8.2).

\section{Materials, drugs, and nomenclature}

We purchased BTT-3033 (1-(4-fluorophenyl)-N-methyl$\mathrm{N}-[4[[($ phenylamino)carbonyl $]$ amino $]$ phenyl $]-1 \mathrm{H}$-pyrazole4-sulfonamide), a small molecule inhibitor with assumed selectivity for integrin $\alpha 2 \beta 1$ heterodimers, from Tocris (Bristol, UK). Stock solutions $(10 \mathrm{mM})$ were prepared in DMSO, and stored at $-20^{\circ} \mathrm{C}$ until used.

\section{Statistical analysis}

The quantitation of protein or phosphate sites between three groups were statistically analyzed by Kruskal-Wallis test and posthoc using R. $\mathrm{P}<0.05$ was defined as significance, were used to screen the differentially expressed proteins or differentially modified phosphate sites.

\section{Results}

\section{Effects of BTT-3033 on the viability of WPMY-1 cells}

To examine the effects of BTT-3033 on stromal proliferation, we assessed the effects of $0.3,1,3,10 \mu \mathrm{M}$ 
A

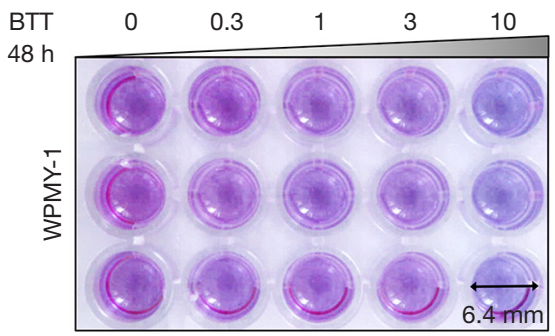

B

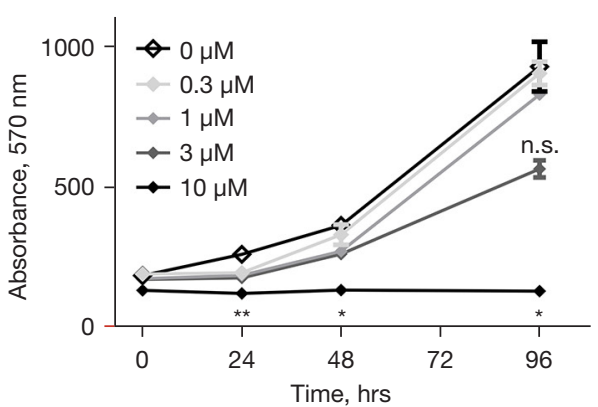

Figure 1 Viability of WPMY-1 cells after BTT-3033 treatment. (A) Representative picture of cell viability by the alamarBlue assay at 48 hrs. alamarBlue fluoresces and changes color in response to chemical reduction to reflect cell viability by the degree of the conversion. When cellular activity is high, alamarBlue is metabolically converted to a pink derivative. (B) WMPY-1 cells were analyzed by an alamarBlue assay at different time points after the application of BTT-3033 $(0.3$ or $10 \mu \mathrm{M})$ or DMSO. Lines with distinct node shapes represent different concentrations of BTT-3033. Ongoing cell proliferation is shown by absorbance at $570 \mathrm{~nm}$. For the time-concentration viability curves, data are presented as mean \pm standard error of the mean $(\mathrm{SEM})$ from $\mathrm{n}=3$ experiments for each concentration and timepoint. ${ }^{* *}$ represents significant $(0.001 \leq \mathrm{P}$ value $<0.01)$, ${ }^{*}$ represents significant $(0.01 \leq \mathrm{P}$ value $<0.05$, and $\mathrm{n}$.s. represents not significant $(\mathrm{P}$ value $\geq 0.05$. $)$.

of BTT-3033 on the viability of WPMY-1 cells using alamarBlue assays (see Figure 1). 0.3 and $1 \mu \mathrm{M}$ of BTT3033 did not alter the viability of the WMPY-1 cells within $96 \mathrm{~h}$; while $3 \mu \mathrm{M}$ of BTT-3033 compromised their viability at the 96-h timepoint (although without significance). The cells did not show any sign of proliferation with $10 \mu \mathrm{M}$ of BTT-3033. Thus, while $1 \mu \mathrm{M}$ of BTT-3033 inhibited tissue contraction, it did not inhibit proliferation. Conversely, $10 \mu \mathrm{M}$ BTT-3033, stopped the cell growth. Thus, BTT3033 was shown to have an inhibitory effect on contraction and proliferation at low $(1 \mu M)$ and high $(10 \mu M)$ concentrations, respectively.

\section{Prediction of co-regulated proteins with integrin a2ß1}

To better understand the mechanism underlying BTT3033 , the possible downstream targets or pathways with relationships to integrin $\alpha 2 \beta 1$ were predicted using the bioinformatic tool ProteomeHD (15). The top 100 proteins co-regulated with ITGA2 were identified (see Table S1) and enriched in KEGG pathways, and the GO biological processes were analyzed thereafter (see Figure $2 A, 2 B$ ). Not surprisingly, integrin $\beta 1$ is among the top co-regulated proteins, along with myosin light chain kinase (MYLK), which is the key kinase to increase smooth muscle tone. The top enriched KEGG pathway and GO biological processes were the "regulation of actin cytoskeleton" and "small GTPase mediated signal transduction," respectively. Consequently, BTT-3033 appeared to inhibit contraction by mediating alterations of the actin cytoskeleton and
GTPase signaling; however, it should be noted that these findings require further verification.

\section{Effects of 1 and $10 \mu M$ of BTT-3033 on protein expression profiles}

To verify the prediction and systematically identify real alterations of BTT-3033, a bottom-up proteomic analysis was conducted on WPMY-1 cells incubated with 1 or $10 \mu \mathrm{M}$ BTT-3033 or DMSO for 6 hours (see Figure 3A). The sample quality of the cell lysates was confirmed to be "excellent" (see Figure S1). The proteomic clustering analysis recognized proteins with similar expression patterns among three groups and classified them into 6 clusters, which are presented as parallel coordinate plots (see Figure 3B). Proteins downregulated by BTT-3033 treatment were allocated into Clusters 2 and 5. The annotation groups in these two enriched clusters were mainly involved in mitosis, cell cycle, cell division, and centrosome (see Figure 3C). Notably, proteins associated with integrins or focal adhesion did not exhibit significant changes. Thus, there was only limited evidence that BTT-3033 reduces contraction by affecting protein expression levels.

\section{Protein phosphorylation alterations by 1 and $10 \mu M$ of BTT-3033 using phosphoproteomic analysis}

Other than protein expression level, phosphorylation is one of the most important posttranslational modifications of proteins that is profoundly involved in the regulation of cell 
A
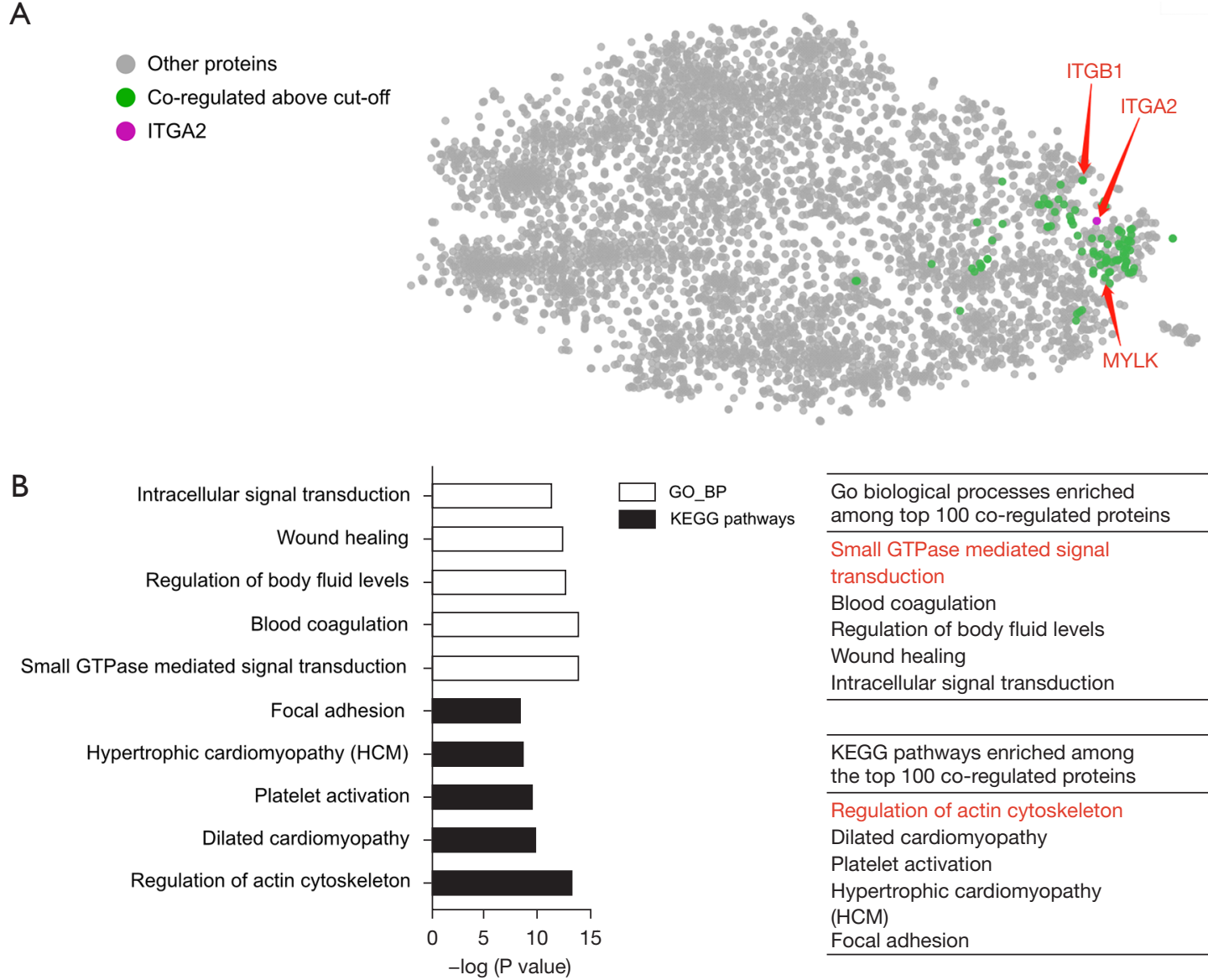

\begin{tabular}{lcc}
\hline $\begin{array}{l}\text { Go biological processes enriched } \\
\text { among top } 100 \text { co-regulated proteins }\end{array}$ & $\begin{array}{c}\text { No. of } \\
\text { proteins }\end{array}$ & $\begin{array}{c}\text { Adj P value } \\
\text { (Bonferroni) }\end{array}$ \\
\hline $\begin{array}{l}\text { Small GTPase mediated signal } \\
\text { transduction }\end{array}$ & 27 & $9.06 \mathrm{E}-15$ \\
Blood coagulation & 25 & $9.68 \mathrm{E}-15$ \\
Regulation of body fluid levels & 26 & $1.63 \mathrm{E}-13$ \\
Wound healing & 26 & $2.21 \mathrm{E}-13$ \\
Intracellular signal transduction & 37 & $3.21 \mathrm{E}-12$ \\
\hline & & \\
\hline KEGG pathways enriched among & No. of & Adj P value \\
the top 100 co-regulated proteins & proteins & (Bonferroni) \\
\hline Regulation of actin cytoskeleton & 17 & $3.64 \mathrm{E}-14$ \\
Dilated cardiomyopathy & 11 & $1.03 \mathrm{E}-10$ \\
Platelet activation & 12 & $2.30 \mathrm{E}-10$ \\
Hypertrophic cardiomyopathy & & \\
(HCM) & 10 & $1.65 \mathrm{E}-09$ \\
Focal adhesion & 13 & $3.72 \mathrm{E}-09$ \\
\hline
\end{tabular}

Figure 2 Proteins co-regulated with integrin alpha-2 (ITGA2). (A) Each dot represents a protein in the proteome-wide map. The purplemarked dot represents ITGA2 and the green-marked dots represent the top 100 proteins co-regulated with ITGA2 of the proteasome that were calculated using ProteomeHD (cutoff score: 0.803824). (B) Left: Bar diagram of the Kyoto Encyclopedia of Genes and Genomes (KEGG) pathways and gene ontology (GO) biological processes enriched among the top 100 co-regulated proteins. Right: KEGG pathways and GO biological processes enriched tables among the top 100 co-regulated proteins; ranked according to the adjusted $\mathrm{P}$ value (Bonferroni).

functions. Thus, the samples used in the proteomic analysis were simultaneously subjected to a phosphoproteomic analysis (see Figure 4A). The clustering analysis of the phosphorylated proteomics suggested that inhibited modified phosphate sites could be classified into two clusters (i.e., Clusters 5 and 6; see Figure 4B). Proteins with phosphate sites that were inhibited in a dose-dependent manner were classified into Cluster 6 that enriched in GO analysis including "actin binding", and annotation including "LIM domain" (see Figure 4C,4D). Notably, we had previously reported the effects of LIM kinase inhibitors on contractions of human prostate strips and the actin organization of WPMY-1 cells, and LIM kinase inhibitors showed a similar inhibition pattern as that of BTT-3033 (see discussion). In relation to enrichment, LIM domain and actin-binding protein 1 (LIMA1), thyroid receptor-interacting protein 6 (TRIP6), and zyxin (ZYX) were suggested to be able to functionally connect focal adhesion and cytoskeleton (see Figure 4E). Thus, among the downstream proteins of focal adhesion which regulate the cytoskeleton, 3 LIM domain proteins (i.e., LIMA1, TRIP6, and ZYX) were detected to have gradient-inhibited phosphate sites.

\section{Kinase-substrate enrichment analysis}

In addition to the individual phosphorylation level, the KSEA used the mean $\log 2$ (FC) of known phosphosite substrates of a given kinase to systematically calculate kinase activity (16). A KSEA was conducted using the phosphoproteomic data, and it was suggested that the kinase 

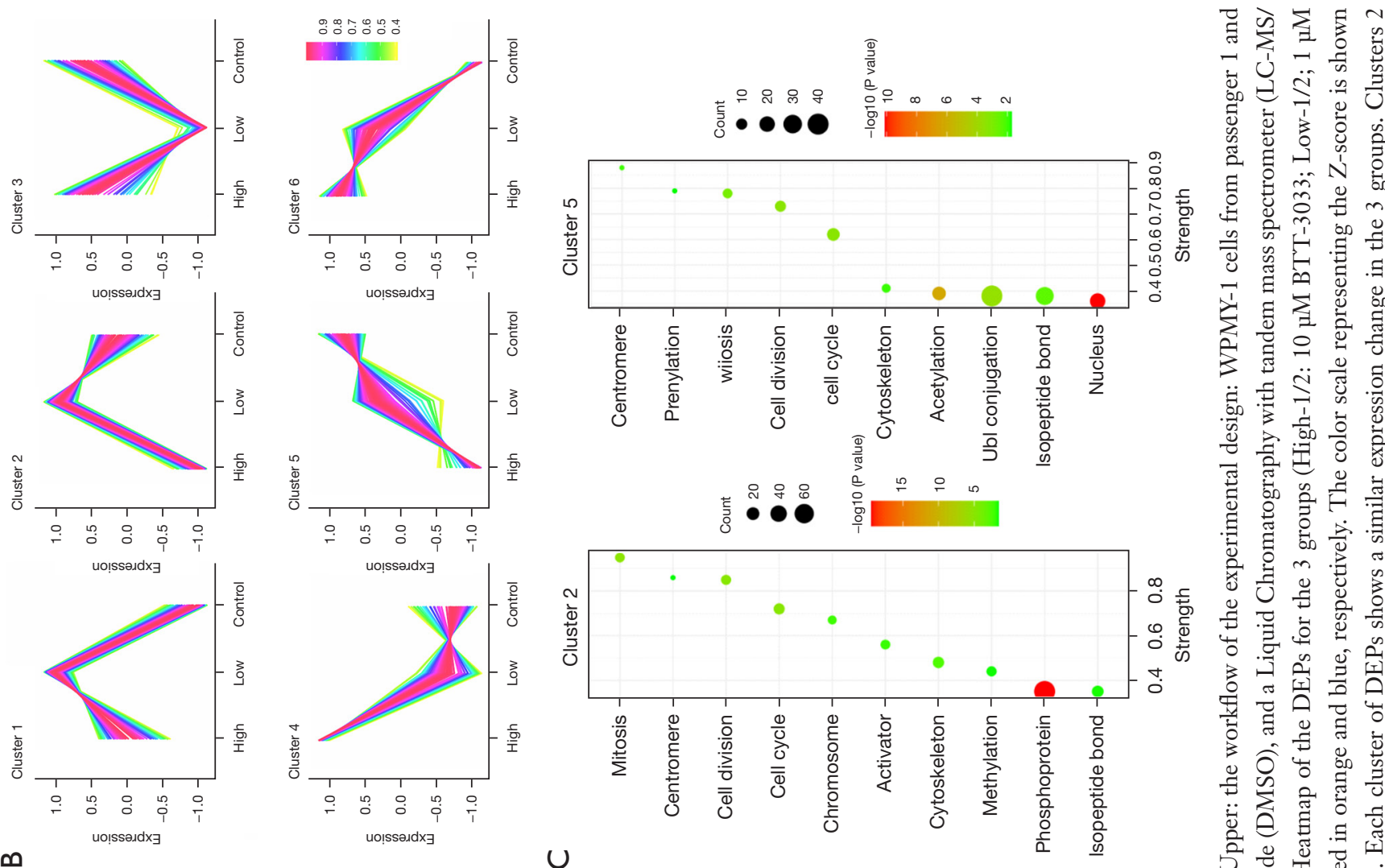

$\infty$
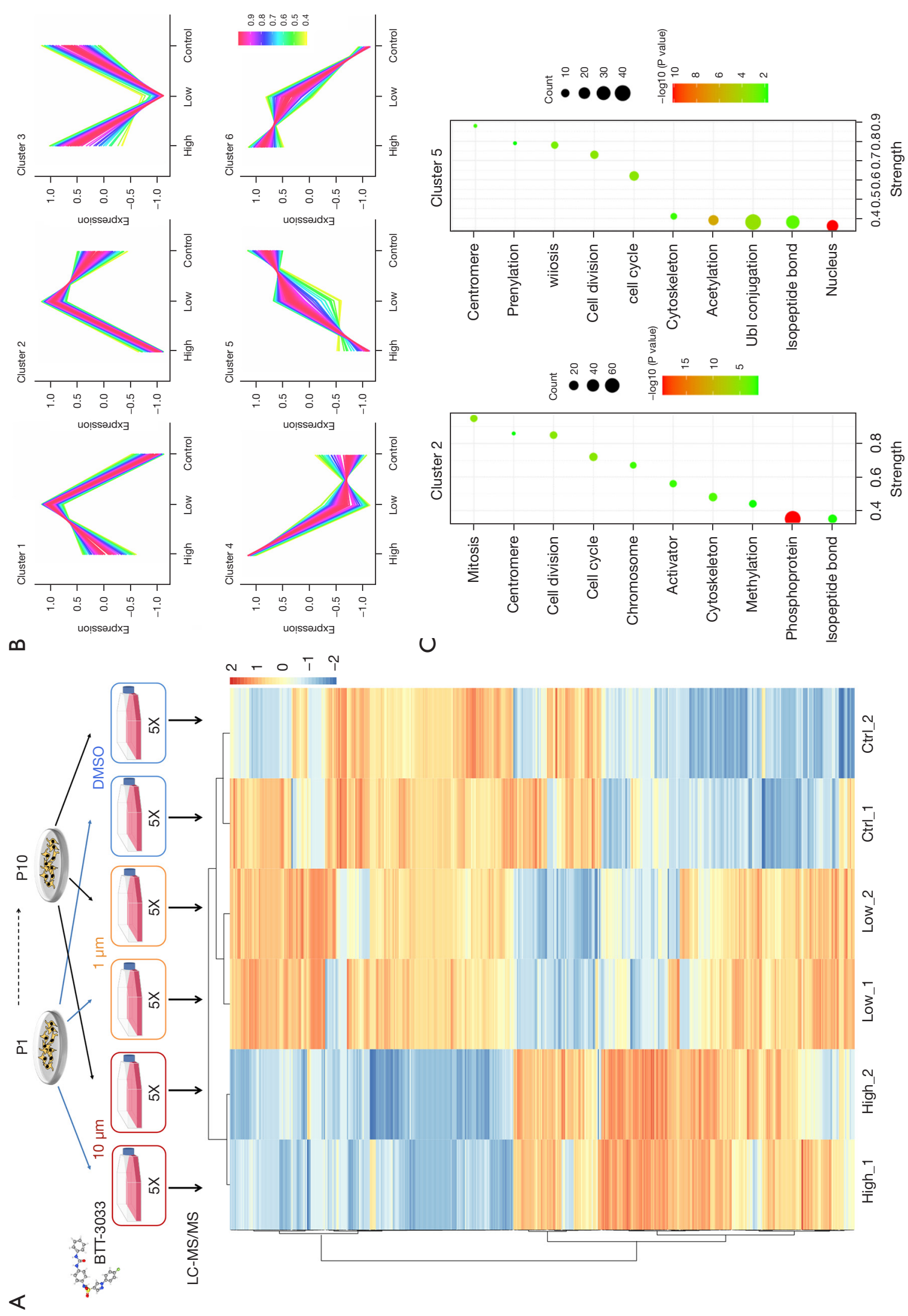

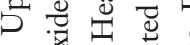

ङิ

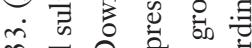

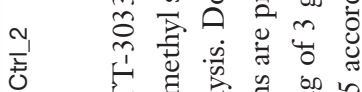

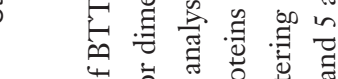

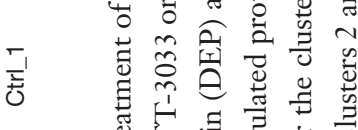

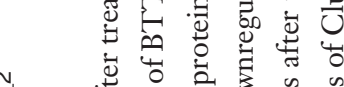

资

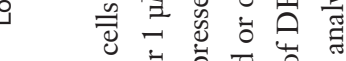

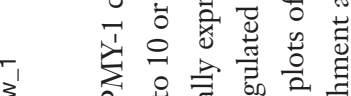

उ

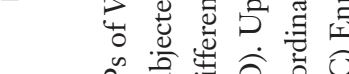

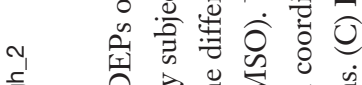

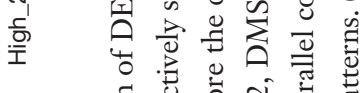

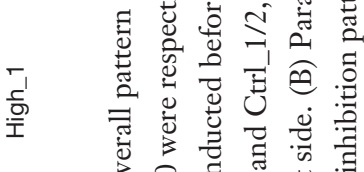

b응

$m$ 茄

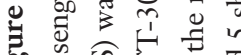

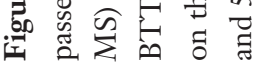


A
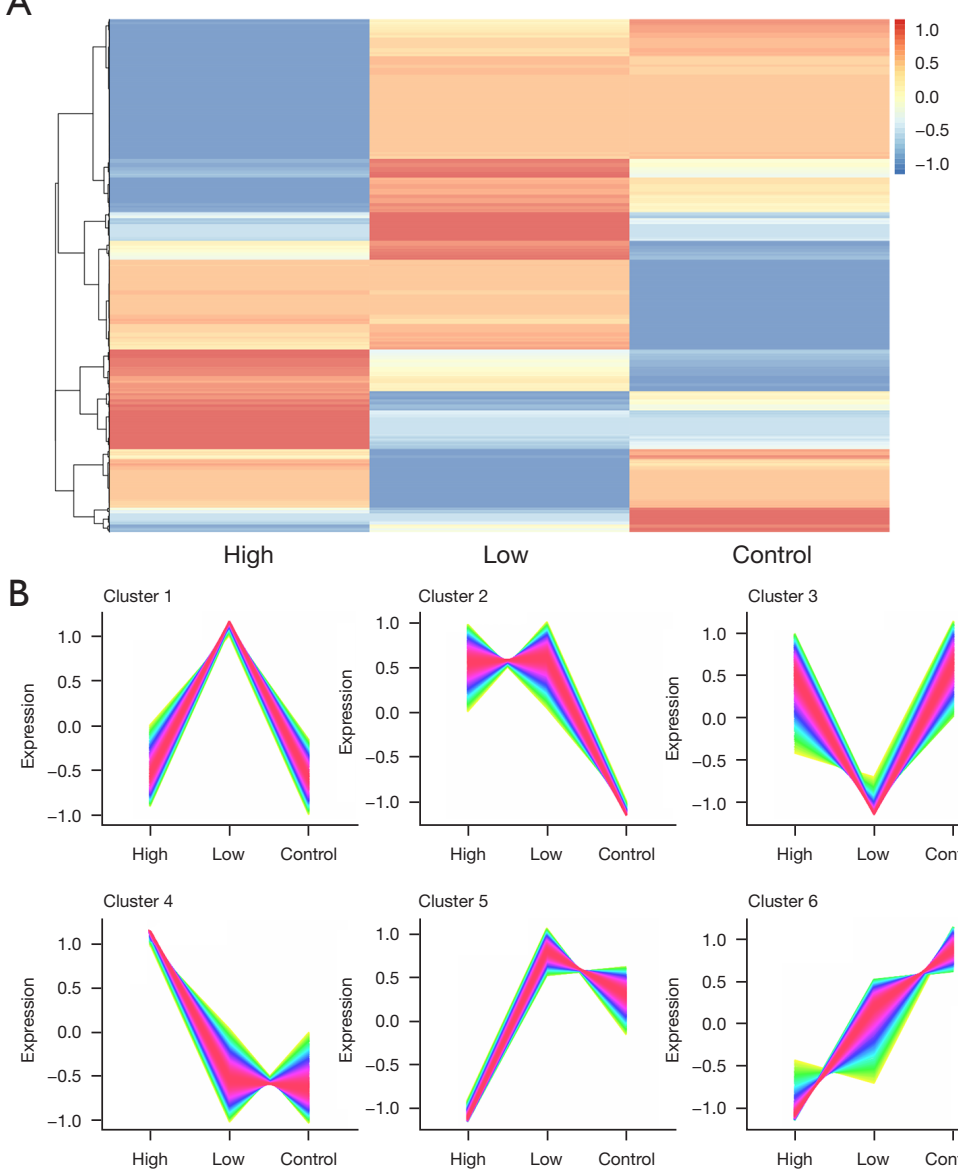

Cluster 2

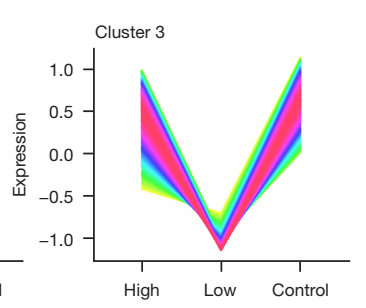

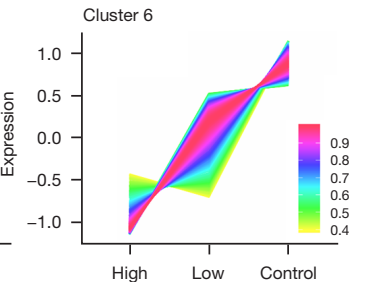

C

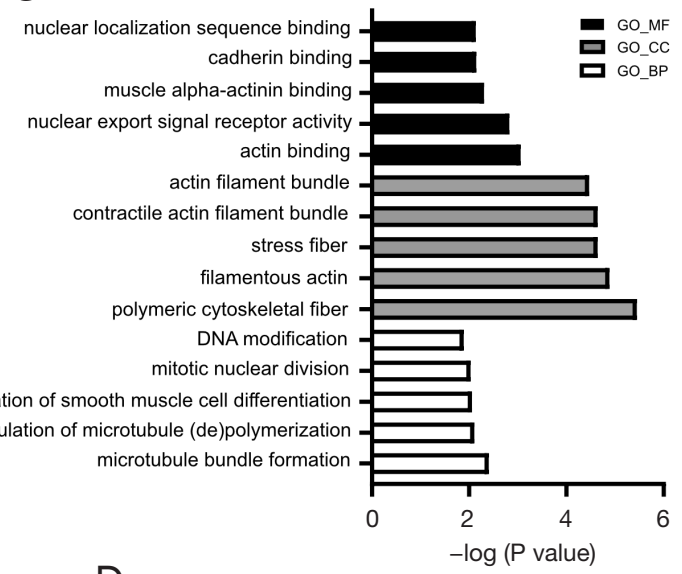

D

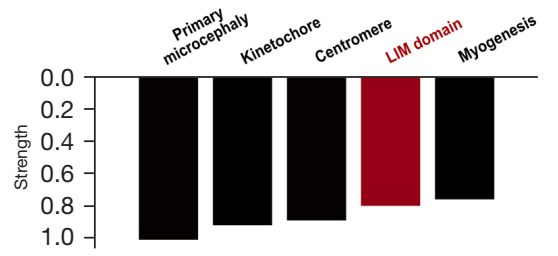

$\mathrm{E}$

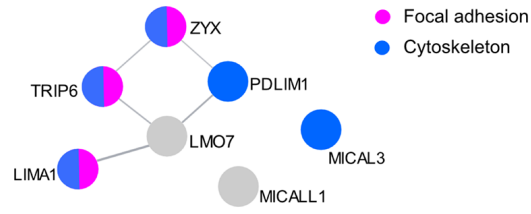

Figure 4 Phosphoproteomic analysis, and GO and annotation enrichment of WPMY-1 cells after BTT-3033 treatment. (A) Heatmap of differentially detected phosphorylated sites for the three groups (high: $10 \mu \mathrm{M}$ of BTT-3033; low: $1 \mu \mathrm{M}$ of BTT-3033, and Ctrl: DMSO). Upregulated or downregulated phospho-sites are presented in red and blue, respectively. The color scale representing the Z-score is shown on the right side. (B) Parallel coordinate plots of DEPs after the clustering of three groups. Each cluster of the DEPs shows a similar expression change in the three groups. Cluster 6 shows a gradient inhibition pattern. (C) Gene ontology (GO) enrichment of phosphoproteomic results. Black-GO enrichment of molecular function. Grey-GO enrichment of cellular component. White-GO enrichment of biological process. (D) Annotation enrichment of Cluster 6. (E) PPI analysis for the "LIM domain" enrichment. Violet dots and blue dots represent proteins for focal adhesion and the cytoskeleton, respectively.

substrates in the GSK3, ACTR2/ACTR2B/TGFbR2, TTK, AuroraA, and CDK2/CDK3/CDK1/CDK5 groups were deactivated by BTT-3033 (starting at $1 \mu \mathrm{M}$ ) (see Figure $5 A$ ). Specifically, disks large homolog 1 (DLG1), cell division cycle 25B (CDC25B), disheveled segment polarity protein 2 (DVL2), myocyte-specific enhancer factor 2A (MEF2A), GLI family zinc finger 2 (GLI2), ABL proto-oncogene 1 (ABL1), centrosomal protein of $192 \mathrm{kDa}$ (CEP192), nuclear factor of activated T-cells 5 (NFAT5), nucleophosmin (NPM1), polo-like kinase 1 (PLK1), E3 ubiquitin-protein ligase NEDD4-like (NEDD4L), nuclear mitotic apparatus protein 1 (NUMA1), brain-specific angiogenesis inhibitor 1-associated protein 2 (BAIAP2), sperm-associated antigen 5 (SPAG5), and serine/threonine-protein kinase N2 (PKN2) are the substrates for which BTT-3033 gradually inhibits phosphorylation (see Figure 5B). A String network showed their correlations, and enrichment occurred in the Rho GTPase binding, cytoskeletal protein binding, and cell cycle (see Figure 5C). Notably, the PPI analysis showed that DVL2, a protein in the Rho GTPase signaling pathway, was able to establish a connection between cytoskeletal pathway proteins PLK1 and ABL1 (see discussion). 

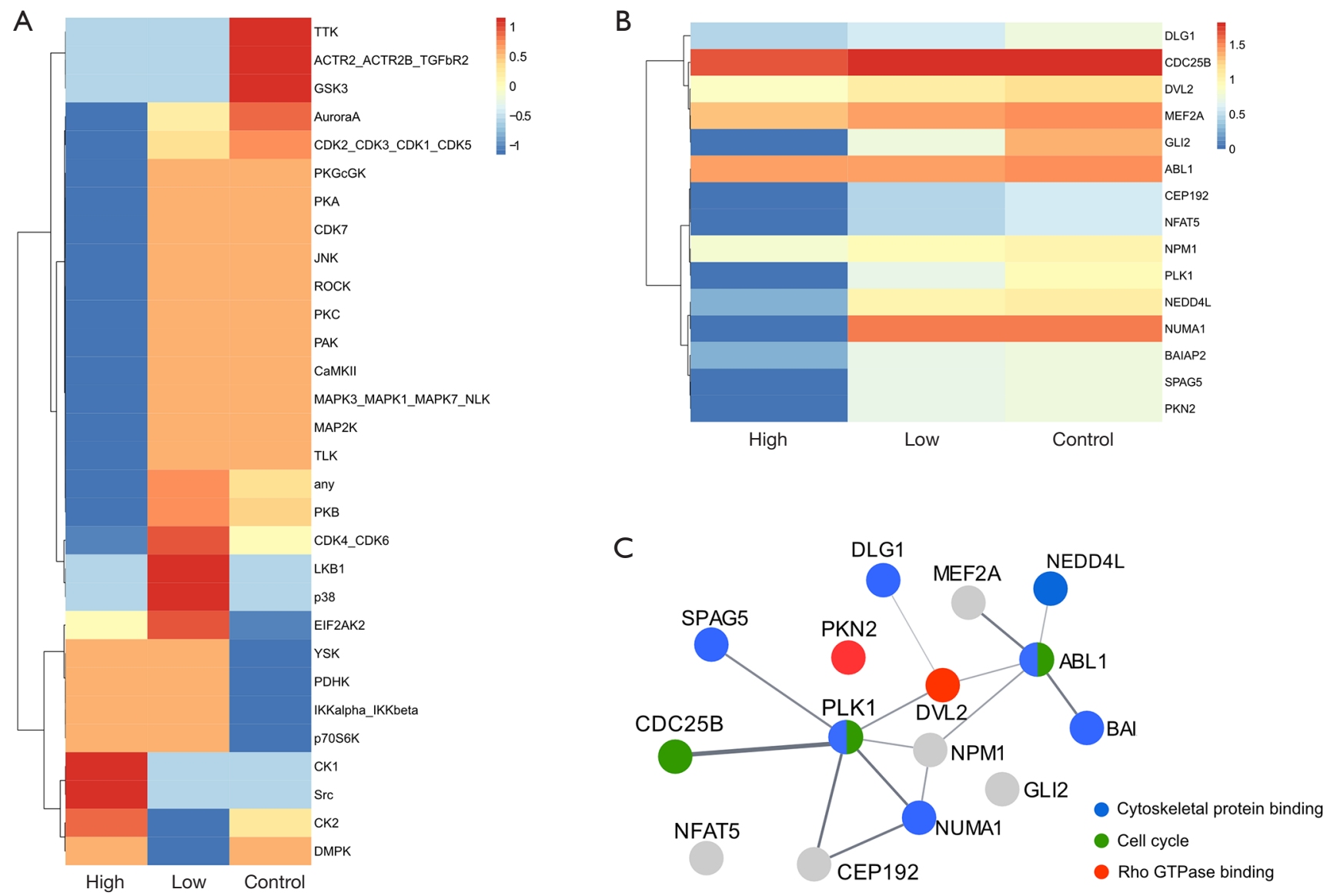

Figure 5 The KSEA and PPI analysis of substrates were predicted to be gradually inhibited by BTT-3033. (A) Alterations of kinasesubstrate relationships in categories according to kinase-substrate enrichment analysis (KSEA). (B) The individual substrates predicted to be gradient-inhibited by BTT-3033. (C) PPI analysis of gradient-inhibited substrates. The red dots are substrates for Rho guanosine triphosphatase (GTPase) binding; the blue dots are substrates for cytoskeletal protein binding; the green dots are substrates for the cell cycle.

\section{Discussion}

In our previous study, we showed that $1 \mu \mathrm{M}$ of BTT-3033 (an integrin $\alpha 2 \beta 1$ inhibitor) inhibited neurogenic and thromboxane $\mathrm{A}_{2}$-induced contractions of human prostate strips, but did not affect endothelin-1-induced contraction and limited the inhibition of contractions by adrenergic agonists (4). In that study, the cytotoxicity tests suggested that the effect of $1 \mu \mathrm{M}$ of BTT-3033 was reversible after its removal. This is in line with the cell viability assay in this study, which showed that $1 \mu \mathrm{M}$ of BTT-3033 did not compromise the viability of WPMY-1 cells. However, $10 \mu \mathrm{M}$ of BTT-3033 blocked the cell cycle. It is not surprising that an inhibitor in a higher concentration can inhibit proliferation given that it can suppress contraction, and that functions related to cytoskeleton motility are fundamental to most cell processes, including mitosis and ribosome action (19-21). Proliferation inhibition is a molecular strategy for reducing stromal growth in the treatment of BPH, and if a potential medication component could be identified that affects contraction and proliferation at different concentrations, more accurate functional modulation could result. $10 \mu \mathrm{M}$ of BTT-3033, however, has been reported to have effects on platelet function, cardiac ventricular restriction, articular cartilage organization, and other biological processes; however, $1 \mu \mathrm{M}$ of BTT-3033 is less likely to have side effects on other cells $(6,22-24)$. Consequently, the mechanisms by which BTT-3033 affects prostate contraction are the main focus of this discussion.

The cytoplasmic parts of integrins form FA complexes 
with other FA components. FAs are dynamic attachment points for the cytoskeleton at the membrane, mediate cytoskeletal organization, and thus regulate contractility (4). SFK, FAK, and ILK are among the most investigated kinases, are structurally or functionally linked to the FA complex, and have all been shown to be involved in the contraction regulation of prostate tissues $(4,25,26)$. However, our preliminary test (data not shown) indicated their expressions and phosphorylation were unaffected by $1 \mu \mathrm{M}$ of BTT-3033. As these results were inconsistent with our previous assumption, subsequent investigations were conducted. Specifically, we used proteomeHD to predict the top proteins co-regulated with ITGA2. Unsurprisingly, ITGB 1 and MYLK were among the top co-regulated proteins, and the "regulation of actin cytoskeleton" was the leading KEGG pathway enrichment, which supported our previous findings on the effects of the integrin $\alpha 2 \beta 1$ inhibitor BTT-3033. Conversely, "small GTPase mediated signal transduction" was the leading GO biological processes, which suggests that BTT-3033 inhibits contraction by mediating alterations of the actin cytoskeleton and GTPase signaling. Overall, proteomeHD reflects protein co-regulation patterns, but does not focus on specific cells or conditions. Thus, to better understand how BTT-3033 inhibits the contraction of prostate strips, a bottom-up (phospho-)proteomic analysis was conducted to explore possible downstream targets.

In relation to contraction inhibition, $1 \mu \mathrm{M}$ of BTT-3033 was of greater interest than $10 \mu \mathrm{M}$ of BTT-3033 in the context of growth impediment. However, we still conducted an analysis of three groups (i.e., the control group, the 1 $\mu \mathrm{M}$ BTT-3033 group and the $10 \mu \mathrm{M}$ BTT-3033 group) for two reasons. First, it was thought that synchronously downregulated proteins/phosphate sites in both the 1 and $10 \mu \mathrm{M}$ BTT-3033 groups could corroborate each other and improve specificity. Second, it was thought proteins/ sites gradually downregulated by gradient BTT- 3033 could be the most direct downstream targets and could connect contractivity with the cell cycle.

According to the cluster analysis of the phosphoproteomics, several enrichments are in line with patterns of synchronous and gradual inhibition. Among these, the "LIM domain" caught our attention, as we had previously reported that the inhibitors for LIM kinases reduced human prostate smooth muscle contraction ex vivo (the human prostate strip) and in vitro (WPMY-1 cells) (14). Additionally, LIMA1, ZYX, and TRIP6 were shown to be related not only to the "cytoskeleton," which is essential to contractivity, but also to "focal adhesion," which is more directly affected by BTT-3033. We assumed that LIMA1, ZYX, and TRIP6, as downstream molecules of integrin $\alpha 2 \beta 1$ and FAs, regulate contractivity by modulating the cytoskeleton. The LIMA1 protein consists of two actin-binding domains separated by a central LIM domain. LIMA1 has been shown to be regulated by focal adhesion complex (9) and to modulate cell dynamics by spatiotemporally interacting with actin (27).

ZYX is a zinc-finger protein with a dual function. An N-terminal domain mediates its association to focal adhesions, and a C-terminal LIM domain mediates proteinprotein and/or protein-deoxyribonucleic acid interactions. ZYX coordinates the organization of actin filaments at focal adhesions, and modulates gene expression in vascular smooth muscle cells by translocating to the nucleus (28). TRIP6, also known as ZYX-related protein-1 (ZRP-1), is an adaptor protein that belongs to the ZYX family of LIM proteins. TRIP6 is primarily localized in the cytosol or focal adhesion plaques, and may be associated with the actin cytoskeleton. Additionally, it is capable of shuttling to the nucleus to serve as a transcriptional coregulator (29).

LIMA1, ZYX, and TRIP6 have not been reported to mediate prostate contraction. LIM kinase 1 and 2 were not (technically) detected in the phosphoproteomic analysis. However, the inhibition pattern of the LIM kinase 1 and 2 inhibitors shares some common ground with that of BTT-3033 in that U46619-induced contractions are most susceptible to the inhibitors and endothelin-1induced contractions are not affected at all. The divergent susceptibility of endothelin-1- and U46619-induced contractions to inhibitors such as BTT-3033 has not yet been well explained. As previously discussed, vascular smooth muscle contractions induced by different agonists may be mediated by different couplings of $G$ proteincoupled receptors to different intracellular effectors. Thus, thromboxane receptors were supposed to predominantly couple with and activate $G \alpha_{12 / 13}$ proteins, while endothelin receptors were supposed to predominantly activate $G \alpha_{q}$ (or $\mathrm{G} \alpha_{\mathrm{q}}$ in addition to $\mathrm{G} \alpha_{12 / 13}$ ). $\mathrm{G} \alpha_{12 / 13}$ activation leads to contraction (mainly via the small GTPase RhoA/Rho kinase pathway), and $\mathrm{G} \alpha_{\mathrm{q}}$ activation mainly occurs in a $\mathrm{Ca}^{2+}$ dependent manner. Further research needs to be conducted to show whether these processes are robust and also applies to prostate smooth muscles. Interestingly, the Rho GTPases pathway may partly support this process, as several types of Rho kinases have been shown to affect smooth muscle contraction in the prostate or in other organs, and these are mainly mediated by $\mathrm{G \alpha}_{12 / 13}$ and independent $\mathrm{Ca}^{2+}$. 
Notably, the KSEA showed that PLK1 was the substrate that gradually inhibited phosphate sites by BTT-3033. It has recently been reported that the PLK1 inhibitor onvansertib inhibits proliferation and adrenergic contraction in the prostate; however, a different spectrum to BTT-3033 was used (30). The relationships of PLK1 and Rho GTPases raised our interest for the following reasons: (I) A recent systematic study revealed that focal adhesion is the crucial place for complicated GTPases regulation (31), and RhoA and Rac have been shown to mediate prostate contraction, while various other Rho GTPases have been shown to be related to smooth muscle contractions in different organs (32); and (II) PLK1 can recruit RhoA guanine nucleotide-exchange factor (GEF) Ect2, and MyoGEF, and regulate RhoA activity $(33,34)$, while the Rac/CDC42 down streaming of integrin can activate PAK1, LIM kinases, and Aurora A/PLK1 $(35,36)$. The upstream molecules of PLK1 are not yet known; however, the later also has a withdraw that the results of the (phospho-)proteomic analysis showed that BTT-3033 did not alter the expression or phosphorylation of PAK1.

KSEA suggested that DVL2 and PKN2 were also substrates which were gradually inhibited, and they were found to be related to GTPases in a more direct way. Previously reports showed that DVL2 enhanced the activation of the Rho family GTPases $(37,38)$, and subsequently mediated various cell dynamic functions. DVL2, which is a downstream target of ABL1 (39), formed a complex with PLK1 (40), and consequently connected these two center nodes shown in the PPI analysis. PKN2 is a $\mathrm{Rho} / \mathrm{Rac}$ effector protein that participates in the regulation of cell cycle progression, actin cytoskeleton assembly, cell migration, cell adhesion, tumor cell invasion and transcription (41-43).

In summary, the network of integrin and LIM domain kinases (especially the ZYX family, PLK1, and kinases, such as DVL2 that are involved in GTPases signaling) are interesting and promising in the context of smooth muscle contraction and proliferation, which are clinically highly relevant in the context of $\mathrm{BPH}$ and require further exploration.

The main limitation of this study is related to its sample numbers, as 6 samples may not be sufficient to avoid sampling bias or technique bias. However, any presented value was based on detection ( 0 means being detectable, but the total detection was very close to 0 instead of being not detected). Further, each sample was prepared by mixing 5 technical duplicates. However, three groups (each comprising 2 samples) with gradient concentration changes may have better selectivity than two groups (each comprising 3 samples). If we had selected the proteins/ sites from the intersection of significant inhibitions, and compared a BTT high group to a BTT low group, and a BTT low group to a control group, the results would have been more convincing even with the limited sample numbers. However, as we elected to sacrifice sensitivity to improve the selectivity of the suggested targets, this study may have failed to detect some of the downstream targets.

\section{Conclusions}

Based on the phosphoproteomic analysis, our findings identified some novel candidate effectors that are involved in the BTT3033-sensitive regulation of prostate smooth muscle contraction and stromal growth. Given the involvement of both processes in LUTS suggestive of BPH, these effectors have high clinical relevance. Future research should be conducted on the contraction effects of integrin $\alpha 2 \beta 1$ and should focus on LIM domain kinases, including the ZYX family, and PLK1 or GTPase-related proteins, such as DVL2.

\section{Acknowledgments}

Funding: This study was supported by grants from the National Natural Science Foundation of China [grant number 81770693], the Deutsche Forschungsgemeinschaft [grant number HE 5825/8-1], the Postdoctoral International Exchange Program of China Postdoc Council (OCPC), and the Chinese Scholarship Council [grant number 201706370083 (BL) and 201706270197 (PL)].

\section{Footnote}

Reporting Checklist: The authors have completed the MDAR reporting checklist. Available at https://dx.doi. org/10.21037/atm-21-3194

Data Sharing Statement: Available at https://dx.doi. org/10.21037/atm-21-3194

Conflicts of Interest: All authors have completed the ICMJE uniform disclosure form (available at https://dx.doi. org/10.21037/atm-21-3194). BL, BZ, ZC, YH and XC report funding support from the National Natural Science Foundation of China (the payments were made to the 
institution); BL, QY, RH, RW, YL, and $\mathrm{MH}$ report funding support from the Deutsche Forschungsgemeinschaft (the payments were made to the institution); BL and PL report personal funding support from the Chinese Scholarship Council. The other authors have no conflicts of interest to declare.

Ethical Statement: The authors are accountable for all aspects of the work in ensuring that questions related to the accuracy or integrity of any part of the work are appropriately investigated and resolved.

Open Access Statement: This is an Open Access article distributed in accordance with the Creative Commons Attribution-NonCommercial-NoDerivs 4.0 International License (CC BY-NC-ND 4.0), which permits the noncommercial replication and distribution of the article with the strict proviso that no changes or edits are made and the original work is properly cited (including links to both the formal publication through the relevant DOI and the license). See: https://creativecommons.org/licenses/by-nc-nd/4.0/.

\section{References}

1. Wang B, Zhang S, Sun C, et al. Comparison between a transurethral prostate split and transurethral prostate resection for benign prostatic hyperplasia treatment in a small prostate volume: a prospective controlled study. Ann Transl Med 2020;8:1016.

2. Foster CS. Pathology of benign prostatic hyperplasia. Prostate Suppl 2000;9:4-14.

3. Chen P, Yin J, Guo YM, et al. The expression and functional activities of smooth muscle myosin and nonmuscle myosin isoforms in rat prostate. J Cell Mol Med 2018;22:576-88.

4. Li B, Wang X, Wang R, et al. Inhibition of neurogenic and thromboxane A2-induced human prostate smooth muscle contraction by the integrin $\alpha 2 \beta 1$ inhibitor BTT-3033 and the integrin-linked kinase inhibitor Cpd22. Prostate 2020;80:831-49.

5. Takada Y, Ye X, Simon S, et al. The integrins. Genome Biol 2007;8:215.

6. Salemi Z, Azizi R, Fallahian F, et al. Integrin $\alpha 2 \beta 1$ inhibition attenuates prostate cancer cell proliferation by cell cycle arrest, promoting apoptosis and reducing epithelial-mesenchymal transition. J Cell Physiol 2021;236:4954-65.

7. Naci D, Vuori K, Aoudjit F, et al. Alpha2beta1 integrin in cancer development and chemoresistance. Semin Cancer Biol 2015;35:145-53.

8. Parks WC. What is the alpha2beta1 integrin doing in the epidermis? J Invest Dermatol 2007;127:264-6.

9. Karaköse E, Geiger T, Flynn K, et al. The focal adhesion protein PINCH-1 associates with EPLIN at integrin adhesion sites. J Cell Sci 2015;128:1023-33.

10. Long $X$, Wong CC, Tong L, et al. Peptostreptococcus anaerobius promotes colorectal carcinogenesis and modulates tumour immunity. Nat Microbiol 2019;4:2319-30.

11. Laplante P, Raymond MA, Labelle A, et al. Perlecan proteolysis induces an alpha2beta1 integrin- and Src family kinase-dependent anti-apoptotic pathway in fibroblasts in the absence of focal adhesion kinase activation. J Biol Chem 2006;281:30383-92.

12. Wang $Y$, Kunit T, Ciotkowska A, et al. Inhibition of prostate smooth muscle contraction and prostate stromal cell growth by the inhibitors of Rac, NSC23766 and EHT1864. Br J Pharmacol 2015;172:2905-17.

13. Li T, Xu K, He J, et al. Effects of isocorynoxeine, from Uncaria, on lower urinary tract dysfunction caused by benign prostatic hyperplasia via antagonism of $\alpha 1 \mathrm{~A}$ adrenoceptors. Toxicol Appl Pharmacol 2019;376:95-106.

14. Yu Q, Gratzke C, Wang Y, et al. Inhibition of human prostate smooth muscle contraction by the LIM kinase inhibitors, SR7826 and LIMKi3. Br J Pharmacol 2018;175:2077-96.

15. Kustatscher G, Grabowski P, Schrader TA, et al. Coregulation map of the human proteome enables identification of protein functions. Nat Biotechnol 2019;37:1361-71.

16. Wiredja DD, Koyutürk M, Chance MR, et al. The KSEA App: a web-based tool for kinase activity inference from quantitative phosphoproteomics. Bioinformatics 2017;33:3489-91.

17. Kolde R. Pheatmap: pretty heatmaps. R package version 2012.

18. Szklarczyk D, Gable AL, Nastou KC, et al. The STRING database in 2021: customizable proteinprotein networks, and functional characterization of user-uploaded gene/measurement sets. Nucleic Acids Res 2021;49:D605-12.

19. Heng YW, Koh CG. Actin cytoskeleton dynamics and the cell division cycle. Int J Biochem Cell Biol 2010;42:1622-33.

20. Chen B, Cao D, Chen Z, et al. Estrogen regulates the proliferation and inflammatory expression of primary stromal cell in benign prostatic hyperplasia. Transl Androl Urol 2020;9:322-31. 
21. Luxenburg C, Zaidel-Bar R. From cell shape to cell fate via the cytoskeleton - Insights from the epidermis. Exp Cell Res 2019;378:232-7.

22. Kanamoto $T$, Hikida $M$, Sato $S$, et al. Integrin $\alpha 2 \beta 1$ plays an important role in the interaction between human articular cartilage-derived chondrocytes and atelocollagen gel. Sci Rep 2021;11:1757.

23. Sun M, Ishii R, Okumura K, et al. Experimental Right Ventricular Hypertension Induces Regional $\beta 1$-IntegrinMediated Transduction of Hypertrophic and Profibrotic Right and Left Ventricular Signaling. J Am Heart Assoc 2018;7:007928.

24. Nissinen L, Koivunen J, Käpylä J, et al. Novel $\alpha 2 \beta 1$ integrin inhibitors reveal that integrin binding to collagen under shear stress conditions does not require receptor preactivation. J Biol Chem 2012;287:44694-702.

25. Wang Y, Gratzke C, Tamalunas A, et al. Smooth muscle contraction and growth of stromal cells in the human prostate are both inhibited by the Src family kinase inhibitors, AZM475271 and PP2. Br J Pharmacol 2016;173:3342-58.

26. Kunit T, Gratzke C, Schreiber A, et al. Inhibition of smooth muscle force generation by focal adhesion kinase inhibitors in the hyperplastic human prostate. Am J Physiol Renal Physiol 2014;307:F823-32.

27. Taha M, Aldirawi M, März S, et al. EPLIN- $\alpha$ and $-\beta$ Isoforms Modulate Endothelial Cell Dynamics through a Spatiotemporally Differentiated Interaction with Actin. Cell Rep 2019;29:1010-1026.e6.

28. Cattaruzza M, Lattrich C, Hecker M, et al. Focal adhesion protein zyxin is a mechanosensitive modulator of gene expression in vascular smooth muscle cells. Hypertension 2004;43:726-30.

29. Lin VT, Lin FT. TRIP6: an adaptor protein that regulates cell motility, antiapoptotic signaling and transcriptional activity. Cell Signal 2011;23:1691-7.

30. Wang X, Li B, Ciotkowska A, et al. Onvansertib, a pololike kinase 1 inhibitor, inhibits prostate stromal cell growth and prostate smooth muscle contraction, which is additive to inhibition by $\alpha 1$-blockers. Eur J Pharmacol 2020;873:172985.

31. Müller PM, Rademacher J, Bagshaw RD, et al. Systems analysis of RhoGEF and RhoGAP regulatory proteins reveals spatially organized RAC1 signalling from integrin adhesions. Nat Cell Biol 2020;22:498-511.

32. Li B, Wang R, Wang Y, et al. Regulation of smooth muscle contraction by monomeric non-RhoA GTPases. Br J Pharmacol 2020;177:3865-77.

33. Li J, Wang J, Jiao H, et al. Cytokinesis and cancer: Polo loves ROCK'n' Rho(A). J Genet Genomics 2010;37:159-72.

34. Petronczki M, Glotzer M, Kraut N, et al. Polo-like kinase 1 triggers the initiation of cytokinesis in human cells by promoting recruitment of the RhoGEF Ect2 to the central spindle. Dev Cell 2007;12:713-25.

35. Kichina JV, Goc A, Al-Husein B, et al. PAK1 as a therapeutic target. Expert Opin Ther Targets 2010;14:703-25.

36. Macůrek L, Lindqvist A, Lim D, et al. Polo-like kinase-1 is activated by aurora A to promote checkpoint recovery. Nature 2008;455:119-23.

37. Habas R, Dawid IB, He X, et al. Coactivation of Rac and Rho by Wnt/Frizzled signaling is required for vertebrate gastrulation. Genes Dev 2003;17:295-309.

38. Zhu Y, Tian Y, Du J, et al. Dvl2-dependent activation of Daam1 and RhoA regulates Wnt5a-induced breast cancer cell migration. PLoS One 2012;7:e37823.

39. Matsumura S, Hamasaki M, Yamamoto T, et al. ABL1 regulates spindle orientation in adherent cells and mammalian skin. Nat Commun 2012;3:626.

40. Xie J, Han M, Zhang M, et al. PP5 (PPP5C) is a phosphatase of Dvl2. Sci Rep 2018;8:2715.

41. Vincent S, Settleman J. The PRK2 kinase is a potential effector target of both Rho and Rac GTPases and regulates actin cytoskeletal organization. Mol Cell Biol 1997;17:2247-56.

42. Schmidt A, Durgan J, Magalhaes A, et al. Rho GTPases regulate $\mathrm{PRK} 2 / \mathrm{PKN} 2$ to control entry into mitosis and exit from cytokinesis. EMBO J 2007;26:1624-36.

43. Morissette MR, Sah VP, Glembotski CC, et al. The Rho effector, PKN, regulates ANF gene transcription in cardiomyocytes through a serum response element. Am J Physiol Heart Circ Physiol 2000;278:H1769-74.

(English Language Editor: L. Huleatt)

Cite this article as: $\mathrm{Li} \mathrm{B}, \mathrm{Li}$ P, Xia W, You B, Yu Q, Zhang B, Huang R, Wang R, Liu Y, Chen Z, Gan Y, He Y, Hennenberg M, Stief CG, Chen X. Phosphoproteomics identifies potential downstream targets of the integrin $\alpha 2 \beta 1$ inhibitor BTT-3033 in prostate stromal cells. Ann Transl Med 2021;9(17):1380. doi: 10.21037/atm-21-3194 
Table S1 Top 100 proteins co-regulated with ITGA2

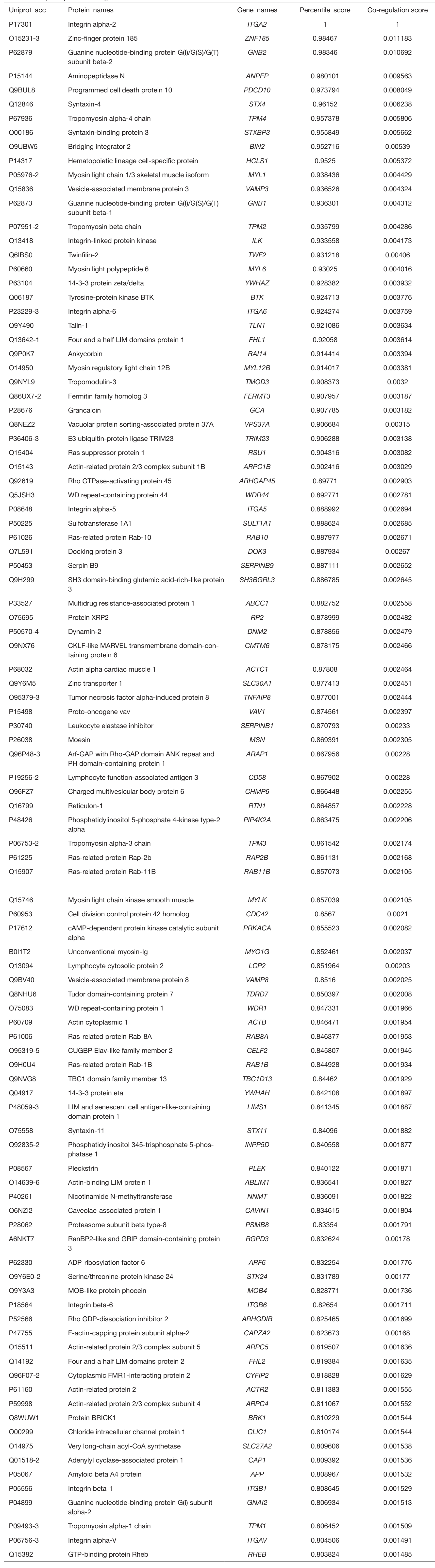



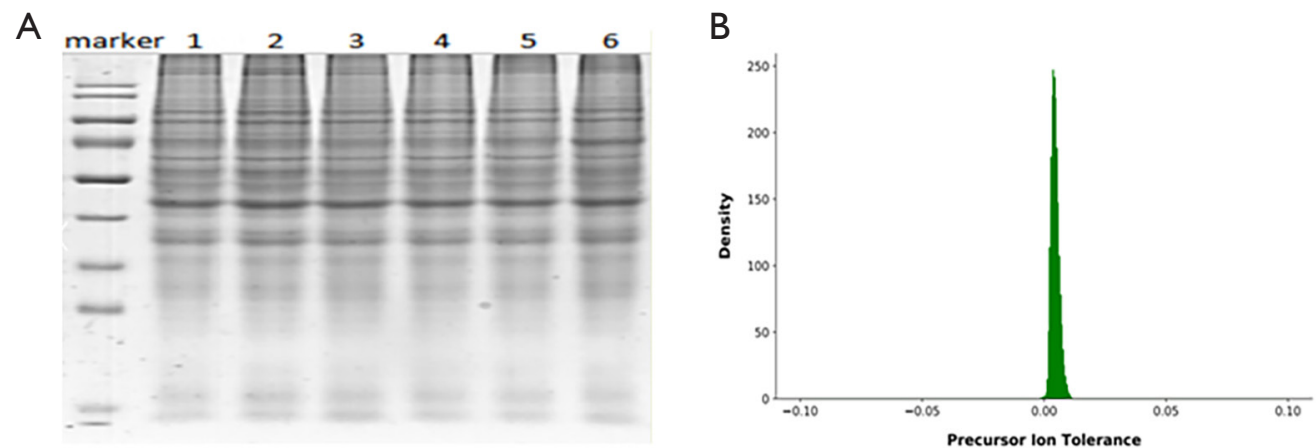

Figure S1 Assessment of the sample quality and the system reliability for (phospho)-proteomic analysis. (A) The sodium dodecyl sulphatepolyacrylamide gel electrophoresis (SDS-PAGE) gel with 6 samples presented matching degree of the protein concentration and molecular weight gradient and indicated an excellent sample quality. (B) According to the mass tolerance distribution of the precursor ion, the peak shape is concentrated close to 0 and indicated the mass spectrometer error mass deviation is small. 